\title{
CYCLOTRON MODELING PHASE-RESOLVED INFRARED SPECTROSCOPY OF POLARS. III. AM HERCULIS AND ST LEO MINORIS
}

\author{
Ryan K. Campbell ${ }^{1,2}$ and Thomas E. Harrison ${ }^{1,3}$ \\ Astronomy Department, New Mexico State University, Las Cruces, NM 88003 \\ AND \\ STELLA KAFKA \\ Spitzer Science Center, Mail Stop 220, California Institute of Technology, \\ 1200 East California Boulevard, Pasadena, CA 91125 \\ Received 2008 March 18; accepted 2008 April 9
}

\begin{abstract}
We present phase-resolved low-resolution infrared spectra of AM Her and ST LMi, two low-field polars that we observed with SPEX on the IRTF. Optical/NIR light curves are also published to help constrain the viewing geometry and brightness of the objects at the time they were observed. Currently, only limited IR spectra have been published for these objects, and none with the phase-coverage presented here. In both cases, the resulting spectra are dominated by emission from the secondary star in the NIR. However, the emission regions are also self-eclipsed, allowing us to isolate the cyclotron emission through subtraction of the dim-phase spectrum. We use a constant-lambda prescription to model the changing cyclotron features seen in the resulting data. For AM Her, we find a best-fit model of $B=$ 13.6 MG, $k T=4.0 \mathrm{keV}$, and $\log \Lambda=5.0$. The cyclotron derived accretion geometry is consistent with $i=50^{\circ}$ and $\beta=85^{\circ}$. For ST LMi, $B=12.1 \mathrm{MG}, k T=3.3 \mathrm{keV}$, and $\log \Lambda=5.7$, with $i=55^{\circ}$ and $\beta=128^{\circ}$.
\end{abstract}

Subject headings: novae, cataclysmic variables

\section{INTRODUCTION}

Polars are interacting binary systems containing a primary white dwarf(WD) and a late-type secondary star. Material flows from the secondary, through the $L_{1}$ point, and falls ballistically toward the WD. The WDs in polars are highly magnetized with magnetic field strengths that range between 10 and 240 MG. Eventually the accreting material couples to the field lines of the $\mathrm{WD}$ and is transported to the magnetic pole(s) of the star, where a dense, standing shock is formed, with nominal temperatures of $2-20 \mathrm{keV}$, which cools by emitting bremsstrahlung and cyclotron radiation.

AM Her is the prototype polar (see Tapia 1977). It has an orbital period of $3.094 \mathrm{hr}$ and is nearby ( $78 \mathrm{pc}$; Thorensten et al. 2003). Despite being extensively studied, many characteristics of the system remain uncertain. One point of discrepancy is the exact mass of the primary $\mathrm{WD}$, with estimates ranging from $0.39 M_{\odot}$ (Young et al. 1981) to $1.22 M_{\odot}$ (Cropper et al. 1998). In addition, the geometry of the system remains unclear, with orbital inclination estimates ranging from $i=35^{\circ}$ (Brainerd \& Lamb 1985) to $i=60^{\circ}-80^{\circ}$ (Watson et al. 2003), although the self-eclipse observed in the $\mathrm{X}$-ray and $\mathrm{UV}$ requires that $i+\beta \geq 90^{\circ}$, where $\beta$ is the magnetic colatitude. Indeed, both Sirk \& Howell (1998) and Gänsicke et al. (1998) found that $i+\beta=105^{\circ}$. Later Gänsicke et al. (2001) determined that combinations of $i$ and $\beta$ between $\left(i=50^{\circ}, \beta=55^{\circ}\right)$ and $\left(i=35^{\circ}, \beta=70^{\circ}\right)$ best modeled the high-state optical light curves. In other ways, however, AM Her is well characterized. The temperature of the primary WD has been well constrained. Gänsicke et al. (2006) modeled low-state Far Ultraviolet Spectroscopic Explorer (FUSE) and Space Tele-

\footnotetext{
1 Visiting astronomer at the Infrared Telescope Facility (IRTF), which is operated by the University of Hawaii under a cooperative agreement with the National Aeronautics and Space Administration.

2 Supported by New Mexico State Space Grant.

3 Visiting Astronomer, Kitt Peak National Observatory.
}

scope Imaging Spectrograph (STIS) spectra from AM Her with TLUSTY/SYNSPEC (Hubeny \& Lanz 1995), finding that $T_{\mathrm{wd}}=$ $19,800 \pm 700 \mathrm{~K}$. Also, the secondary star is spectroscopically determined to be M4 \pm 1 (Kafka et al. 2005), although there is some evidence that it is irradiated by the WD, so that the spectral type of the secondary is orbitally modulated (Davey \& Smith 1992). The published photometry of AM Her is exhaustive. Orbital light curves show variability in every passband from the UV (Gänsicke et al. 1998) out to the $K$-band (see below). Extensive AAVSO and automatic photometric telescope (APT; e.g., RoboScope) monitoring from 1990 to 2004, has revealed that AM Her is usually in one of two states: a "high state," with large intrinsic variability $(13.0 \leq V \leq 14.0)$ and a "low state," with $V \simeq 15.5$ (Kafka et al. 2005). Finally, Bailey et al. (1991; hereafter BFW91) used a constant-lambda (CL) code to model the NIR low state $(V \sim 15.0)$ cyclotron spectrum, which were binned into bright-phase $(\phi=0.46-0.88)$ and dim-phase $(\phi=$ $0.46-0.88)$ spectra. The dim phase showed only emission from the secondary star, while strong cyclotron emission was observed for the duration of the bright phase. After subtracting a $3250 \mathrm{~K}$ model atmosphere with $\log g=4.75$ to mimic the secondary spectrum, BFW91 found $B \simeq 14.5 \mathrm{MG}$ and a shock temperature of $k T=8.5 \mathrm{keV}$.

ST LMi $(=\mathrm{CW} 1103+254)$ is a short-period polar $\left(P_{\text {orb }}=\right.$ 114 minutes) containing a $0.7 M_{\odot}$ primary (Ramsay et al. 2004) with a likely temperature of $11,000 \mathrm{~K}$ (Araujo-Betancor et al. 2005; Sion 1999) and a M5-M6 secondary (Knigge 2006; Harrison et al. 2005; Howell et al. 2000; Warner 1995) at a distance of 115-138 pc (Araujo-Betancor et al. 2005; Kafka et al. 2007). It was classified as an AM Her object by Stockman et al. (1983) on the basis of its highly variable polarization. For $70 \%$ of the orbit the object shows no significant polarization. Subsequently, a strong pulse is observed peaking near $\phi=0.00$ at $12 \%$ and $-20 \%$ in linear and circular light, respectively (Cropper et al. 1986). This observed bimodality is echoed in optical and IR 
TABLE 1

OBSERVING LoG

\begin{tabular}{|c|c|c|c|c|c|}
\hline Date & Object & Instrument & Observation Type & $\begin{array}{c}I \\
(\mathrm{~s})\end{array}$ & State \\
\hline 2005 Sep 1 & AM Her & IRTF SPEX & Spectroscopic & 120 & Low \\
\hline 2002 Sep 26 & AM Her & KPNO & Photometric & & Low \\
\hline 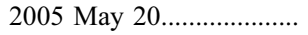 & AM Her & APO & Photometric & 240 & Low \\
\hline 2005 Feb 7 & ST LMi & IRTF SPEX & Spectroscopic & & Extreme low \\
\hline 2006 Feb 2 ........................... & ST LMi & IRTF SPEX & Spectroscopic & & Low \\
\hline 2003 Apr 9................... & ST LMi & KPNO & Photometric & 240 & High \\
\hline
\end{tabular}

orbital light curves, which show a quiescent "dim phase" for most of the orbit in each band that is followed by a significant jump in brightness (the "bright phase") coincident with the peak in polarization. Peacock et al. (1992) obtained multiband photometry of ST LMi, showing that $\Delta m \simeq 0.6,1.4,2.0,1.5$, and 1.3 for the $B R I J H$ bands, respectively. Long-term $V$-band light curves were obtained with RoboScope from 1990 to 2003 (Kafka et al. 2005). From 1992-1997, the system was in a protracted low state with $\langle V\rangle=17.5 \pm 0.2$. From 1997 to 2003 , a more variable, slightly higher state was observed with $\langle V\rangle=16.0 \pm 1.5$. In addition, instances of "extreme low states" have been observed. In Ciardi et al. (1998) the $K$-band spectra of ST LMi, showed no obvious emission lines and were modeled successfully with $\mathrm{a} \simeq 3000 \mathrm{~K}$ atmosphere, suggesting that accretion had almost completely shut off. Kafka et al. (2007) has presented photometry of a similar extreme low state showing that the system can be as faint as $V=18.5$. JHK cyclotron spectra were previously modeled by Ferrario et al. (1993; hereafter FBW93), who found that two spots were necessary to fully model their spectra: a primary region with $B=12.0$ and $k T=12 \mathrm{keV}$, and a secondary, "cool spot" with $k T=5.0 \mathrm{keV}$. The accretion geometry of the primary emission region has also been previously determined, with $55^{\circ} \leq$ $i \leq 64^{\circ}$ and $140^{\circ} \leq \beta \leq 150^{\circ}$ (Schmidt et al. 1983; Potter 2000).

Limited IR spectroscopy exists for these two low-field polars. Below we present and model new phase-resolved low-state infrared spectra as well as $J H K$ light curves for AM Her and ST LMi. In both instances, we show that variable cyclotron emission over the orbit is responsible for the spectroscopic and photometric behavior. In addition, we present a second-epoch data set for ST LMi that shows no cyclotron emission and must be in an extreme low state, similar to that seen by Kafka et al. (2007). In the next section we describe the observations of each object, in $\S 3$ we fit these data with cyclotron models; we discuss our results in $\S 4$ and draw our conclusions in $\S 5$.

\section{OBSERVATIONS}

AM Her and ST LMi were observed using SPEX (Rayner et al. 2003) on the Infrared Telescope Facility (IRTF). AM Her was observed once on 2005 September 1, whereas ST LMi was observed on two different epochs: 2005 February 7 and 2006 February 2. Both AM Her and ST LMi were found to be in low states, although as we discuss below, the 2005 February 7 data found ST LMi in an extreme low state. SPEX was used in low-resolution prism mode with a $0.3^{\prime \prime} \times 15^{\prime \prime}$ slit. To remove background, each object was nodded along the slit. In its lowresolution mode SPEX produces $R(=\lambda / \Delta \lambda) \sim 250$ spectra, with short enough exposure times to obtain phase resolved spectra of polars with $K \leq 16.0$. For ST LMi, we used 240 s exposure times, where shorter, $120 \mathrm{~s}$, integration times were adequate for AM Her. Each of these spectra were then median combined with two to three other spectra to allow for cosmic-ray removal and to improve the signal-to-noise ratio. The spectra were reduced using the SPEXTOOL package (Vacca et al. 2003). A telluric correction was applied using an $\mathrm{A} 0 \mathrm{~V}$ star of similar air mass to our program objects. We use the Kafka et al. (2005) ephemeris to phase all observations of AM Her. For ST LMi, because of the large phase uncertainty $(\Delta \phi \simeq 0.10)$ in the Howell et al. (2000) ephemeris, we phased our observations to the $J$-band minimum found in the photometry presented in this paper, which worked out to a phase shift of $\Delta \phi=0.15$ from that ephemeris.

Because of the narrow slit size on IRTF SPEX $\left(0.3^{\prime \prime}\right)$ infrared photometry is required to calibrate the fluxes of the spectra. The $J H K$ photometry for each object was obtained with SQIID on the KPNO $2.1 \mathrm{~m}$ telescope (Ellis et al. 1992). AM Her was observed on 2002 September 26, and ST LMi on 2003 April 9. In addition, we obtained simultaneous BVRIJHK photometry on 2005 May 20 for AM Her. The JHK photometry was obtained with NIC$\mathrm{FPS}^{4}$ on the Apache Point $3.5 \mathrm{~m}$, while the optical data set was obtained with the NMSU $1 \mathrm{~m}$ (see Harrison et al. 2003). To aid the reader, we have collated all the observational specifics in Table 1.

\section{MODELING}

To produce our cyclotron models, we use a constant-lambda cyclotron code first developed by Schwope (1990). In Campbell et al. (2008b, hereafter Paper I), we presented a theoretical synopsis of CL modeling which is not be repeated here. The model spectra depend on four global parameters: $B$ (the magnetic field strength), $k T$ (the plasma temperature), $\Theta$ (the viewing angle to the magnetic "pole"), and $\Lambda$ (the size parameter), which is closely tied to the column density along the line of sight through the accretion region. In Paper I we found that we could adequately model the data for EF Eri as cyclotron+WD. In Campbell et al. (2008b, hereafter Paper II) we found that in many polars there are other sources of nonstellar continuum radiation (e.g., bremsstrahlung emission) which contaminate the spectra and need to be taken into account. For each object in Paper II, the accretion column was self-eclipsed. In this case, cyclotron emission is only seen for the part of the orbital cycle when the accretion column is in view (the bright phase) although it is contaminated by other sources. To subtract these away, we assume that the dim phase spectra represent all the additional components of radiation which obfuscate the cyclotron emission. The dim-phase spectrum is then subtracted from the spectra at other phases where accretion column is in view, thus yielding uncontaminated cyclotron spectra over the orbit. We refer to the dim-phase subtraction method as stream-emission subtraction (SE subtraction) for consistency with Schwope et al. (2002). An additional contaminant for the objects in the current work is the irradiated secondary star whose spectral

\footnotetext{
html.
} 

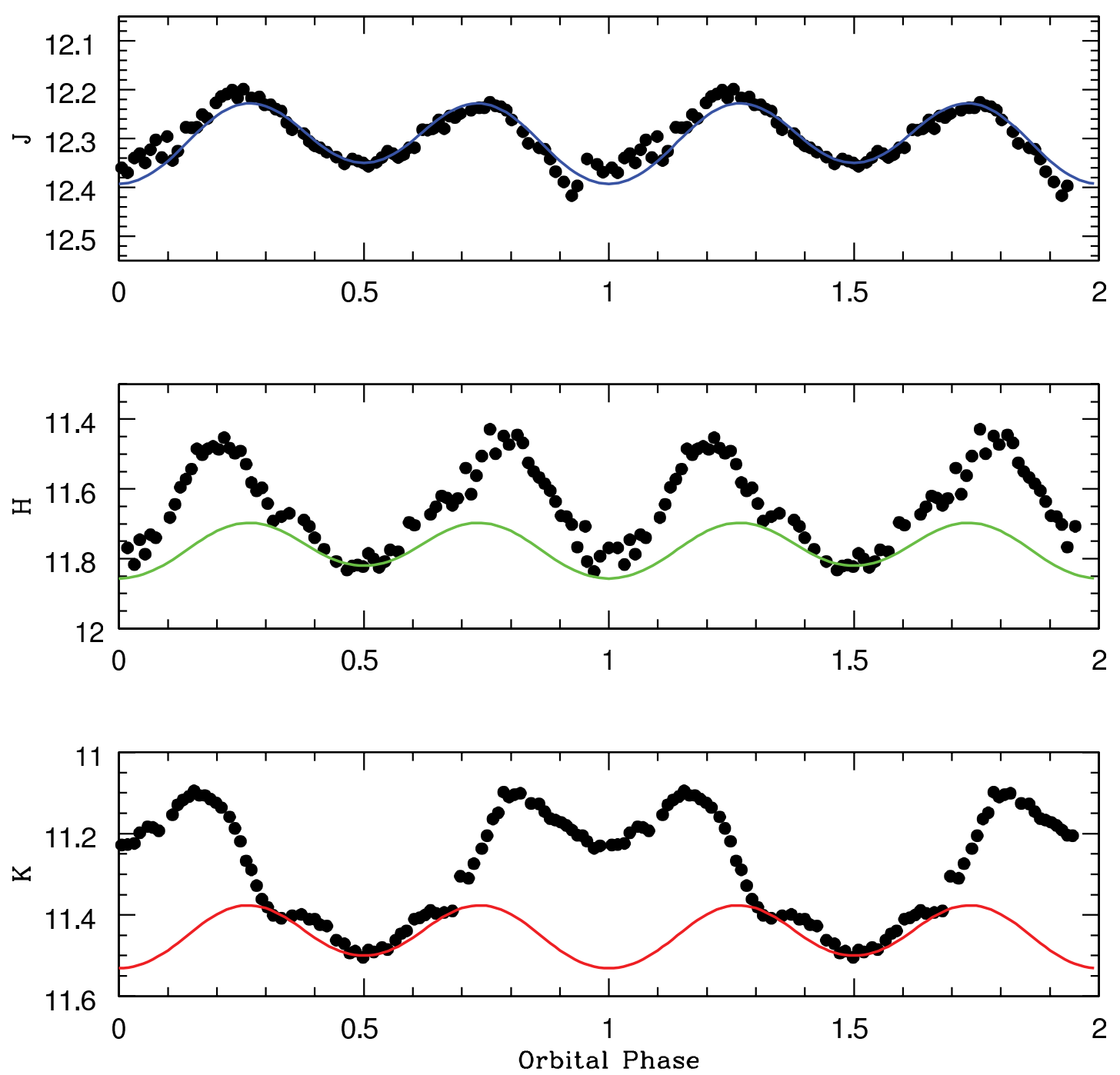

FIG. 1. - JHK photometry of AM Her obtained with the KPNO 2.1-m on 2006 September 26, when the object was in a faint state $(V \sim 15.5)$. The $J$ and $H$ bands show strong ellipsoidal variations, while the $K$-band morphology is the result of a combination of ellipsoidal and cyclotron emission. The light curves were phased using the Kafka et al. (2005) ephemeris. The overplotted lines are ellipsoidal models for $i=50^{\circ}$.

type slowly changes with phase and cannot be completely subtracted. Thus, when the SE subtraction technique was applied features due to the secondary star remained.

\subsection{AM Her}

In Figure 1 we show the $J H K$ light curves taken with the SQIID on the KPNO $2.1 \mathrm{~m}$ on 2002 September 26, at a time when the system was at $V=15.5$. This is a typical low-state magnitude identical to that of our SPEX spectroscopy, as shown in Figure 2. Overlaid in each band are binary star models computed using WD2005 5 with a M5 secondary at an orbital inclination of $i=$ $50^{\circ}$. The $J$-band morphology is well explained by classic ellipsoidal variations, except that the light curve minimum at $\phi=$ 0.00 is somewhat deeper than predicted and residual structure appears at the $\Delta J=0.05 \mathrm{mag}$ level. The derived inclination should be considered a lower limit because other dilution sources may be present. Both the $H+K$ light curves show a large cyclotron component folded in with the ellipsoidal variations. In Figure 3 we include additional $B V R I J H K$ photometry obtained $2.5 \mathrm{yr}$ later,

\footnotetext{
5 WD2005 is an updated version of WD98, and can be obtained at this Web site maintained by J. Kallrath: http://josef-kallrath.orlando.co.nz/HOMEPAGE/ wd2002.htm.
}

on 2005 May 20, at a time when the system was again at similar brightness. We find that the same ellipsoidal models provide excellent fits to the $J H K$ data at this epoch. Because of the narrow slit size on $\operatorname{SPEX}\left(0.3^{\prime \prime}\right)$, at each orbital phase we flux calibrate our spectra to the "cyclotron-free" $J$-band light curve.

The IRTF phase-resolved spectra from 2005 September 1 are dominated by emission from the secondary star. To remove this component, we subtracted the spectrum at $\phi=0.42$ from every other phase. The subtraction spectrum is near to both the ellipsoidal minimum $(\phi=0.50)$ and because of the ongoing selfeclipse, is free of cyclotron emission. To approximate the effect of the ellipsoidal variability, we scaled the subtraction spectrum at each phase to match the magnitude expected from our ellipsoidal models. The underlying continuum SED and intrinsic water vapor features at 1.35 and $1.85 \mu \mathrm{m}$ in the residual spectra were orbitally variable, producing a small blue excess and apparent water vapor emission at ellipsoidal maxima $(\phi=0.25$, $0.75)$ and a red excess with apparent water vapor absorption at ellipsoidal minima $(\phi=0.00,0.50)$, resulting from a changing spectral type as the distorted secondary star changed orientation. From M4 to M6 water vapor absorption becomes ever more pronounced. Thus, even small differences of the secondary temperature are apparent in our data and residuals from the water vapor 

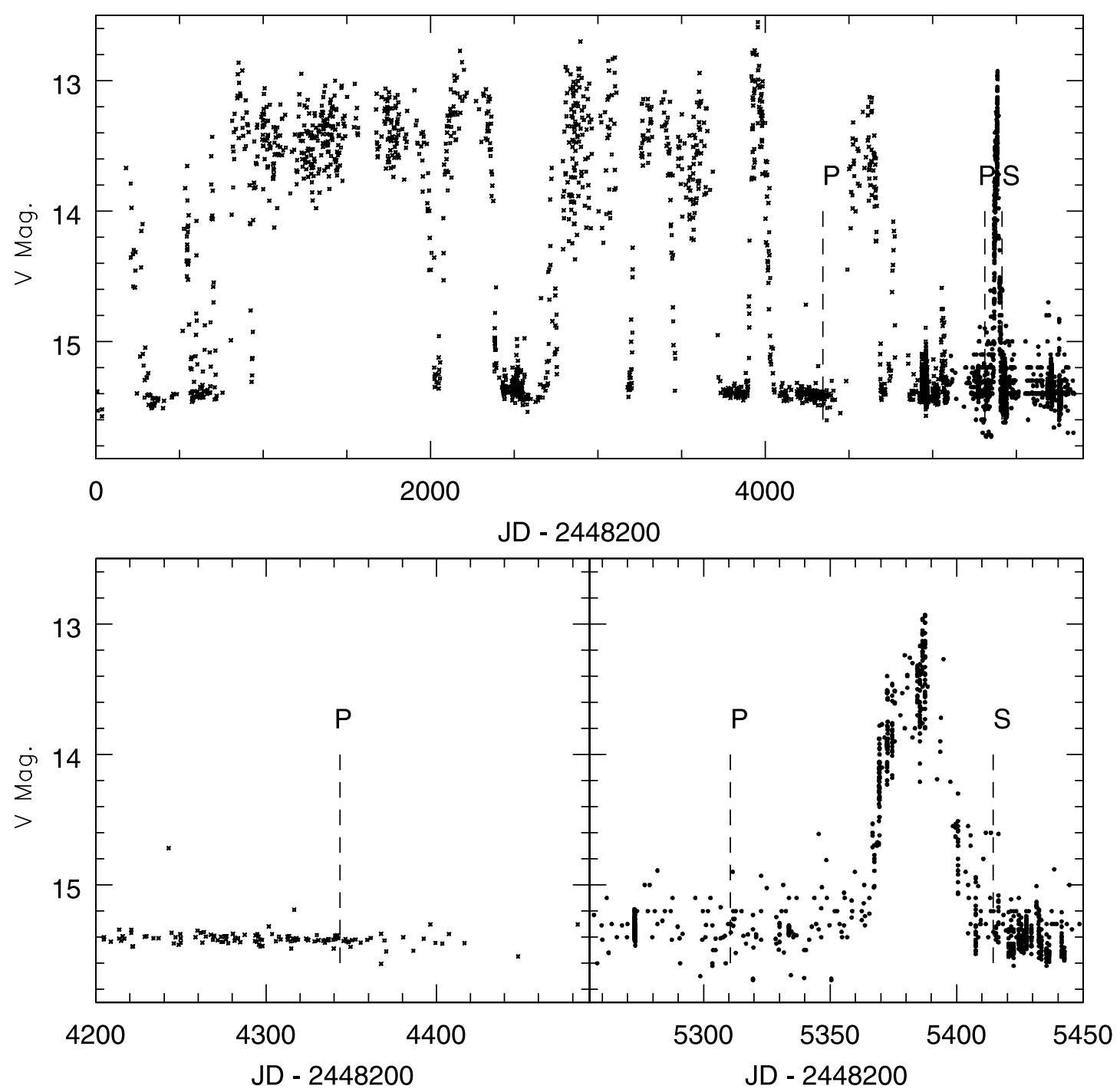

FIG. 2. - Long-term RoboScope light curves of AM Her complimented by AAVSO data. The P denotes the times of our SQIID and NIC-FPS/NMSU $1 \mathrm{~m}$ photometry, while the $\mathrm{S}$ indicates our spectroscopic observations. Top: $V$-band photometry following AM Her from 1991 through 2006. Bottom left: Zoom-in on the year surrounding our SQIID photometric measurements. Bottom right: Zoom-in on the year surrounding our IRTF spectroscopy.

features remain in our final spectra (Fig. 4, in black). The final cyclotron models are overlaid in green. No cyclotron emission was observed over the interval $0.27 \leq \phi \leq 0.74$ due to the selfeclipse of the emission region. Thus, these phases are not shown in Figure 4 to aid in the presentation of data. Between $0.92 \leq$ $\phi \leq 0.09$, the spectra appear to show a single strong cyclotron harmonic $(n=4)$ near $2.0 \mu \mathrm{m}$. The $n=4,5$, and 6 harmonics are obvious between $0.20 \leq \phi \leq 0.26$ and again from $0.75 \leq \phi \leq 0.86$.

For our best-fit cyclotron models at each phase, see Table 2 . The average parameter values are $B=13.6_{-0.8}^{+1.0} \mathrm{MG}, k T=$ $4.0_{-1.0}^{+1.5} \mathrm{keV}$, and $\log \Lambda=5.0 \pm 0.6$, with an average $\chi_{\nu}^{2}=2.42$. The statistical limits were derived by finding where the value of $\chi_{\nu}^{2}$ changed by $50 \%$ over its fiducial value. We find that $i+$ $\beta=135^{\circ}$, with $i=50^{\circ}, \beta=85^{\circ}, \phi_{\min }=0.01$, where $\phi_{\min }$ is defined as the bluest position of the cyclotron harmonics.

\subsection{ST LMi}

In Figure 5 we present $J H K$ light curves of ST LMi taken with the KPNO $2.1 \mathrm{~m}$ during the normal high-accretion state of the system. In each band the morphology is similar: the dim phase lasts from $0.00 \leq \phi \leq 0.55$ with mean magnitudes of 14.4, 13.9, and 13.9 in the $J, H$, and $K$ bands, respectively. During the subsequent bright phase, the object brightens significantly $(\Delta J=$ $1.5 \mathrm{mag}$ ). Like AM Her, a WD2005 ellipsoidal model was fit to the NIR light curves, finding $i=55^{\circ}$. The models well approximate the dim phase of ST LMi in the $J$ and $H$ bands, while the fit in the $K$ band is more uncertain due to the larger scatter in the photometry.

ST LMi was observed spectroscopically over its entire orbit once on 2005 February 7 and once on 2006 February 2. On both occasions, photometry is available within one month of our phaseresolved spectroscopy. Figure 6 shows the high/low states of ST LMi over the entire history of RoboScope and also over a shorter, $\sim 1.5 \mathrm{yr}$ baseline surrounding our 2005 and 2006 IRTF observations. For the first data set, the nearest RoboScope data (February 28) shows $17.1 \leq V \leq 17.8$, similar in brightness to its 1992-1997 protracted low state. However, as we discuss below, our bright-phase spectroscopy shows a conspicuous lack of cyclotron emission during this epoch. For this reason, we believe the object was in fact in an extreme low state at the time of observation, similar to that observed on 2006 February 12 by Kafka et al. (2007), who found $18.0 \leq V \leq 18.4$. Curiously, our 2006 data was obtained only 10 days prior to that epoch, but shows clear 

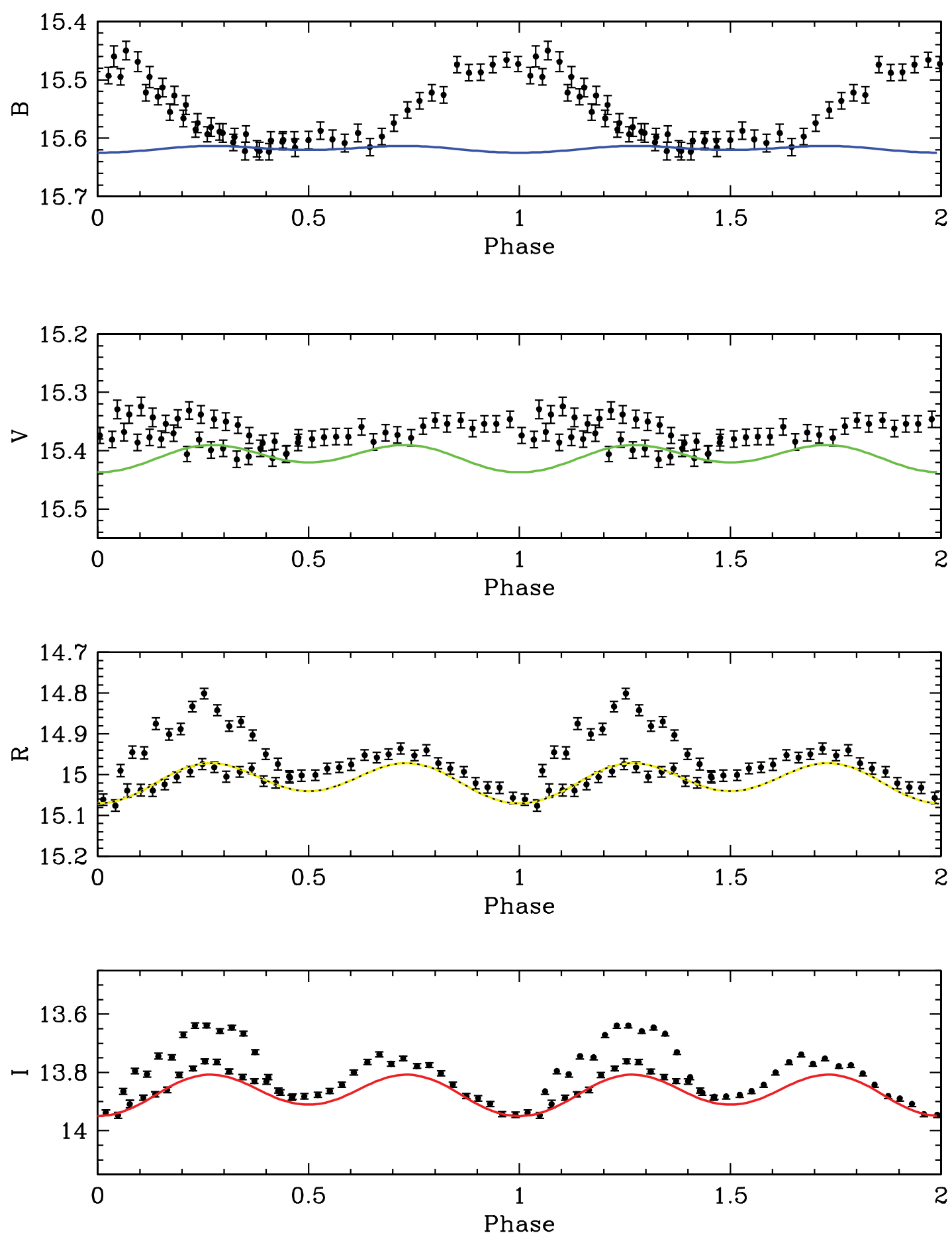

FIG. $3 a$

FIG. 3.- BVRIJHK photometry of AM Her obtained with the APO $3.5 \mathrm{~m} / \mathrm{NMSU} 1 \mathrm{~m}$ on 2005 May 20 when the object was in a faint state $(V=15.3)$ similar to the

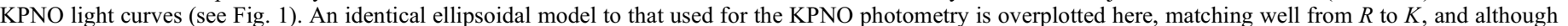
a small flare event is evident in $R$ and $I$ during the second cycle of observation almost no cyclotron emission should be present in these bands. Humps reminiscent of cyclotron emission reappear in the $V$ and especially $B$ bands suggesting that a higher field is active on AM Her. $(a)$ Optical optical bands; $(b)$ NIR bands. 

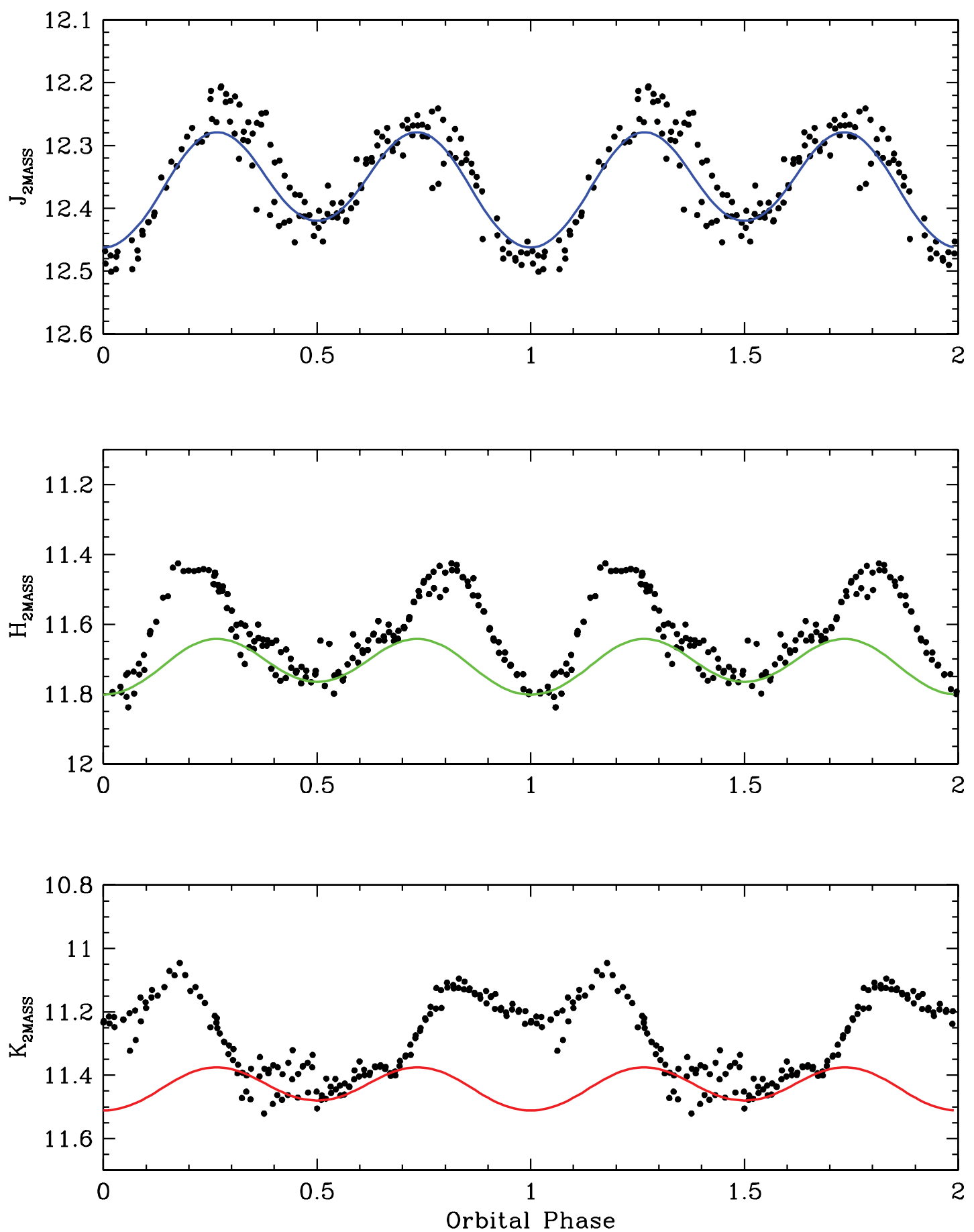

FIG. $3 b$ 


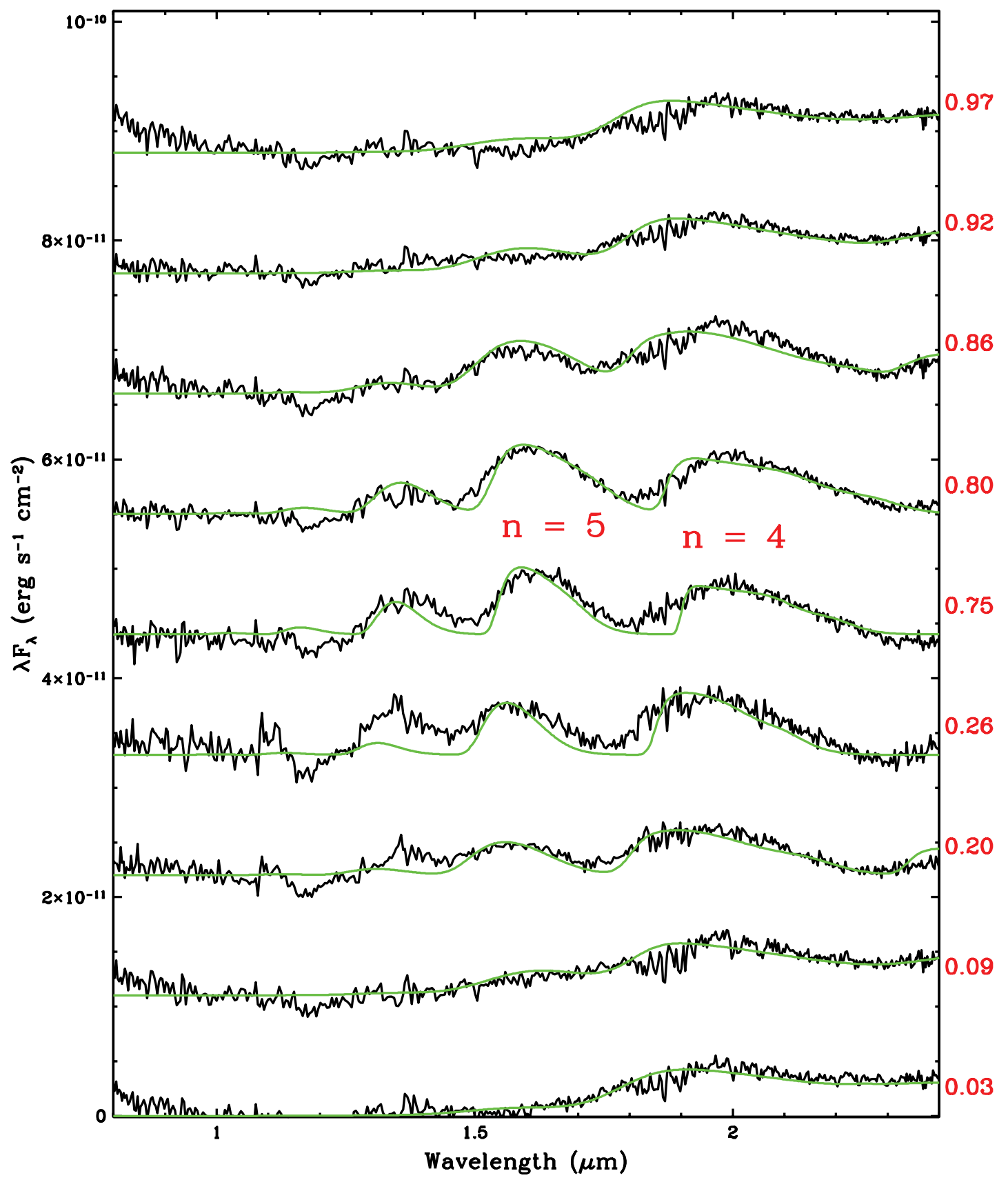

FIG. 4.-IRTF SPEX phase-resolved spectra of AM Her plotted (black) as a stacked series, with a constant flux increment of $\lambda F_{\lambda}=1.10 \times 10^{-11} \mathrm{ergs} \mathrm{s}^{-1} \mathrm{~cm}^{-2}$ and covering the orbital phases for which the cyclotron emission region is in view. At each phase, the $1.22 \mu \mathrm{m}$ flux is normalized to the $J$-band light curve ensuring proper flux calibration with the narrow $0.3^{\prime \prime}$ slit and a dim-phase spectrum $(\phi=0.42)$ was subtracted. Because of the variability of the secondary's spectral type over the orbit, the underlying continuum as well as intrinsic water features changed over the orbit. Remnant intrinsic water features, however, are still apparent at $\sim 1.35$ and $1.8 \mu$ m. No cyclotron emission was observed from $\phi=0.27$ to 0.74 . 
TABLE 2

Cyclotron Modeling Parameters for AM Her

\begin{tabular}{|c|c|c|c|c|c|}
\hline Phase & $\begin{array}{c}B \\
(\mathrm{MG})\end{array}$ & $\begin{array}{c}k T \\
(\mathrm{keV})\end{array}$ & $\theta$ & $\log \Lambda$ & $\chi_{\nu}^{2}$ \\
\hline $0.03 \ldots \ldots \ldots \ldots \ldots$ & 13.3 & 3.9 & 35.0 & 5.3 & 3.53 \\
\hline 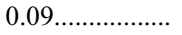 & 13.1 & 4.1 & 42.0 & 5.3 & 3.90 \\
\hline $0.20 \ldots \ldots \ldots \ldots \ldots$ & 14.0 & 3.9 & 62.0 & 4.8 & 1.11 \\
\hline $0.26 \ldots \ldots \ldots \ldots \ldots$ & 14.1 & 3.9 & 72.0 & 4.6 & 2.12 \\
\hline $0.31 \ldots \ldots \ldots \ldots \ldots . .$. & $\ldots$ & $\ldots$ & $\ldots$ & $\ldots$ & $\ldots$ \\
\hline $0.37 \ldots \ldots \ldots \ldots \ldots \ldots$ & $\ldots$ & $\ldots$ & $\ldots$ & $\ldots$ & $\ldots$ \\
\hline $0.48 \ldots \ldots \ldots \ldots \ldots$ & $\ldots$ & $\ldots$ & $\ldots$ & $\ldots$ & $\ldots$ \\
\hline $0.52 \ldots \ldots \ldots \ldots \ldots \ldots$ & $\ldots$ & $\ldots$ & $\ldots$ & $\ldots$ & $\ldots$ \\
\hline $0.59 \ldots \ldots \ldots \ldots \ldots \ldots$ & $\ldots$ & $\ldots$ & $\ldots$ & $\cdots$ & $\ldots$ \\
\hline $0.64 \ldots \ldots \ldots \ldots \ldots \ldots$ & $\cdots$ & $\ldots$ & $\ldots$ & $\cdots$ & $\ldots$ \\
\hline $0.70 \ldots \ldots \ldots \ldots \ldots$ & $\ldots$ & $\ldots$ & $\ldots$ & $\ldots$ & $\ldots$ \\
\hline $0.75 \ldots \ldots \ldots \ldots \ldots$ & 13.8 & 4.3 & 75.0 & 5.0 & 1.78 \\
\hline $0.80 \ldots \ldots \ldots \ldots \ldots$ & 13.6 & 4.3 & 67.0 & 5.2 & 1.30 \\
\hline $0.86 \ldots \ldots \ldots \ldots \ldots \ldots$ & 13.6 & 4.2 & 55.0 & 5.1 & 3.30 \\
\hline $0.92 \ldots \ldots \ldots \ldots \ldots \ldots$ & 13.4 & 4.0 & 46.0 & 5.1 & 2.28 \\
\hline $0.97 \ldots \ldots \ldots \ldots \ldots$ & 13.3 & 3.9 & 37.0 & 5.4 & 2.48 \\
\hline
\end{tabular}

evidence of cyclotron emission and thus must have been in a normal low state. We note, however, photometric extreme low states like that observed by Kafka et al. are short lived, as normal low states were observed within a month both before and after it.

The spectra of ST LMi are also strongly contaminated by its secondary star at every phase. During the extreme low state we found no evidence for cyclotron emission, with the secondary contributing all of the NIR flux at each orbital phase. In Figure 7 we show the observed SPEX spectrum at $\phi=0.02$ of the 2005 data set with the best-fitting secondary template overlaid (M6).

For the 2006 low state, we present SE-subtracted data in Figure $8 a$. The SE spectrum was produced by averaging the dimphase spectra together. Since the relative uncertainty in the Howell et al. (2000) ephemeris is rather large $\left(\Delta \phi_{0} \simeq 0.10\right)$, we phased our data by defining ellipsoidal minimum in our $2003 \mathrm{KPNO}$ light curve as $\phi=0.50$ and then using the Howell et al. (2000) period. We found that averaging three dim-phase spectra together produced the best SE-subtraction spectra, with faint cyclotron features visible during the bright orbital phase at $\sim 2.25,1.85,1.53$, and perhaps $1.30 \mu \mathrm{m}$, corresponding to the $n=4-7$ harmonics in a field with $B=12 \mathrm{MG}$. Like AM Her, the tidally distorted nature of the secondary star imparts spectral type and overall flux changes that are orbitally modulated allowing residual emission/ absorption from the secondary star to remain even after SE subtraction has been performed. Indeed, the upturn in the $J$-band SED as well as the strong water vapor absorption at $\phi \simeq 0.73$ are indicative of subtraction of too cool a secondary star at those phases. In Figure $8 b$, we display the phase-resolved spectra from the 2005 extreme low state. Like the 2006 low state the SE spectrum was found by averaging three dim-phase spectra together. While weak cyclotron emission was observed during the 2006 low state none was seen during the 2005 extreme low state.

Previous work has determined that ST LMi is a one pole accretor with $55^{\circ} \leq i \leq 64^{\circ}$ and $140^{\circ} \leq \beta \leq 150^{\circ}$ (Schmidt et al. 1983; Potter 2000). The primary accretion region also appears to have some structure. FBW93 computed cyclotron models for ST LMi in a high state, finding that two separate emission regions were needed to adequately model the observed spectra from the primary pole: the first is a high-temperature, high-density region located between magnetic longitudes $(\psi) 130$ and 170 that has $k T=12.0 \mathrm{keV}$ and $\log \Lambda=7.6$. The second is located between $170 \leq \psi \leq 250$ with $k T=5 \mathrm{keV}$ and $\log \Lambda=4.4$. Both regions had magnetic field strengths of $\simeq 12.0 \mathrm{MG}$, a result which is consistent with the values previously published. The relevancy of these models to our SPEX data is unclear, since they were determined when the object was in a high state $(J=13.8)$, more similar to that observed in our SQIID photometry than our lowstate spectroscopy. In addition, Peacock et al. (1992) reported seeing a second pole in ST LMi during a high state $(B \sim 16.7)$. Their $H$-band photometry showed that a sudden increase of $\Delta H=0.3$ mag at $\phi=0.35$ was observed, with the $J$-band showing a smaller increase. Simultaneous polarization curves were published along with their photometry that found $V / I=15 \%-$ $20 \%$ over the duration of the alleged secondary pole. The errors, however, were extremely large and the data are in fact, consistent with zero polarization. Because both the phasing and amplitude of the light curve variations are consistent with the WD2005 models found in this study (Fig. 5), we believe the excess $H$-band feature may be due to ellipsoidal variability from the secondary star.

We used the published values $i$ and $\beta$ to constrain the orbital variation of $\Theta$ and thus effectively limit the possible parameter space to three dimensions, $B, k T$, and $\log \Lambda$. Because of the very low rate of accretion, the models presented here have low temperatures. We found $12.0 \leq B(\mathrm{MG}) \leq 12.2,3.2 \leq k T(\mathrm{keV}) \leq$ 3.4 , and $5.5 \leq \log \Lambda \leq 6.1$, and $\Theta$, which is well described with $i=55^{\circ}, \beta=128^{\circ}, \phi_{\max }=0.22 \pm 0.05$. The average $\chi_{\nu}^{2}=$ 2.70. As for AM Her, we find the following uncertainties in the parameter values: $B \pm 0.5 \mathrm{MG}, k T \pm 1.8 \mathrm{keV}$, and $\log \Lambda \pm 0.6$. Table 3 lists the phase-resolved parameters.

\section{DISCUSSION}

\subsection{Analysis of Results}

We have presented the first phase-resolved IR spectroscopy published for these two sources allowing for enhanced leverage over the parameters modeled. The data presented in this paper proved the most challenging to model in this series. In Paper I we covered the basics of cyclotron modeling and applied our technique to NIR spectra of EF Eri finding that it could be modeled using only cyclotron and WD emission. In Paper II we found that other sources of contamination could be eliminated by subtracting the dim-phase spectrum from each phase for which cyclotron emission was observed. In the present work, however, the spectra of both AM Her and ST LMi are dominated by emission from the secondary star in the NIR. Because of the orbitally modulated nature of both the spectral type and brightness of the secondary, additional issues arose. Naive subtraction of only a single dim-phase spectrum from every other phase is manifested in two ways. First, variable water vapor absorption/emission is seen at 1.35 and $1.85 \mu \mathrm{m}$. Second, the underlying SED cycles twice between a red and blue excess over the orbit. Both artifacts result from the changing spectral type of the secondary star. Because we subtract the same phase from each spectrum, the secondary imprint in the SE-spectrum alternates between being too cool and too hot when compared to the features seen at other phases. To assuage the situation, we median combined three dim-phase spectra separated by $\Delta \phi=0.25$, to produce the final SE spectrum, thus smearing out the effects of a changing secondary star.

In AM Her, we found that for the bright-phase of the 2006 low-state $(V \simeq 15.5) \mathrm{SPEX}$ data $B=13.7 \pm 1.0 \mathrm{MG}, k T=$ $4.2 \pm 1.0 \mathrm{keV}$, and $\log \Lambda=5.0 \pm 0.5$. These results are in dramatic contrast with those found in BFW91 at a time when the system was also in a low, although perhaps slightly higher, state $(V \simeq 15.0): B=14.5 \pm 0.3 \mathrm{MG}, k T=8.5 \pm 0.5 \mathrm{keV}$, and $\log \Lambda=3.3 \pm 0.3$. While the magnetic field strengths for the 

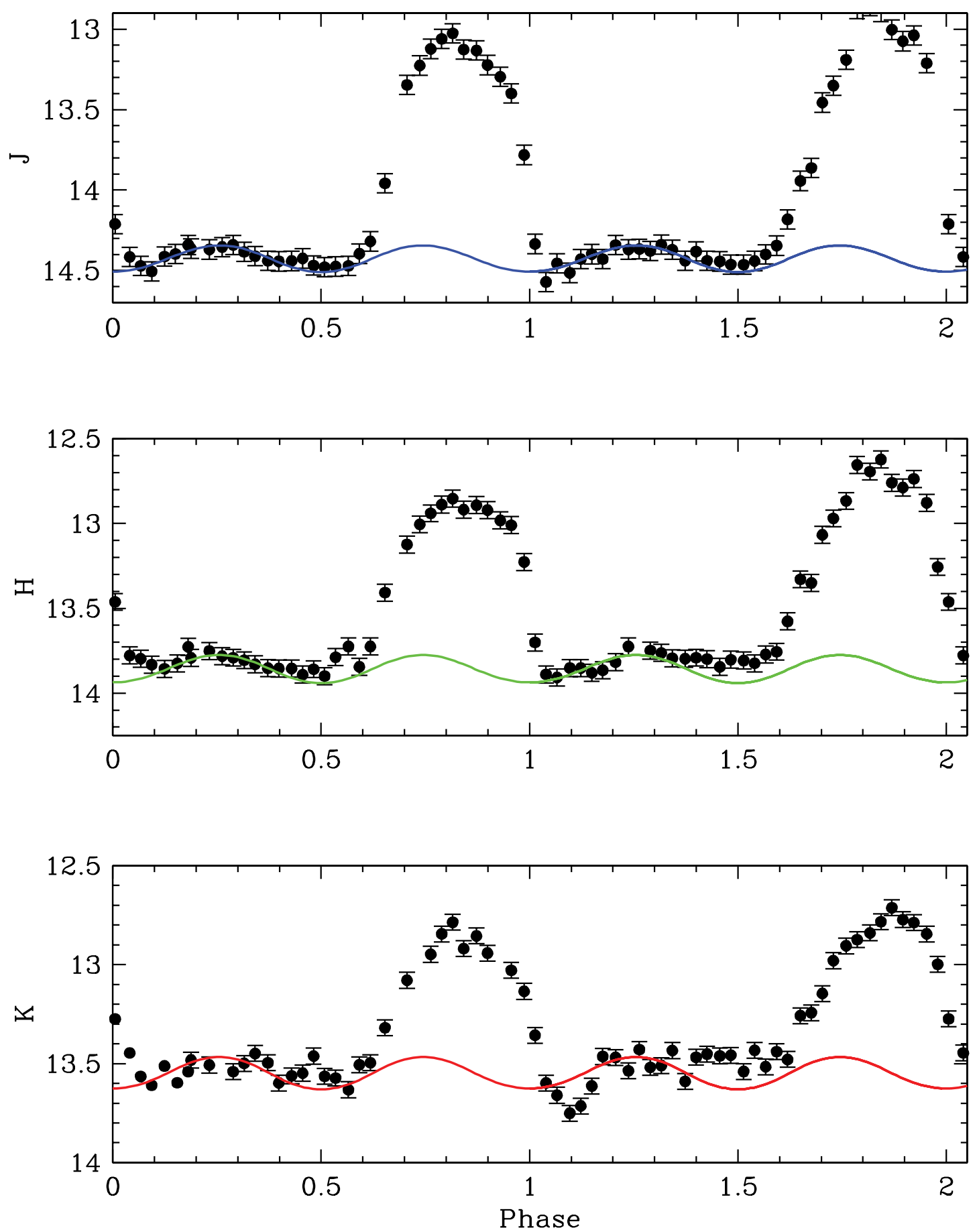

FIG. 5.-JHK photometry of ST LMi obtained with the KPNO $2.1 \mathrm{~m}$ SQIID on the 2003 April 9 high state. The dim-phase lasts from $\phi=0.00$ to 0.55 , while from $\phi=0.60$ to 0.95 the bright-phase is observed. The plotted lines are ellipsoidal models for $i=55^{\circ}$. 

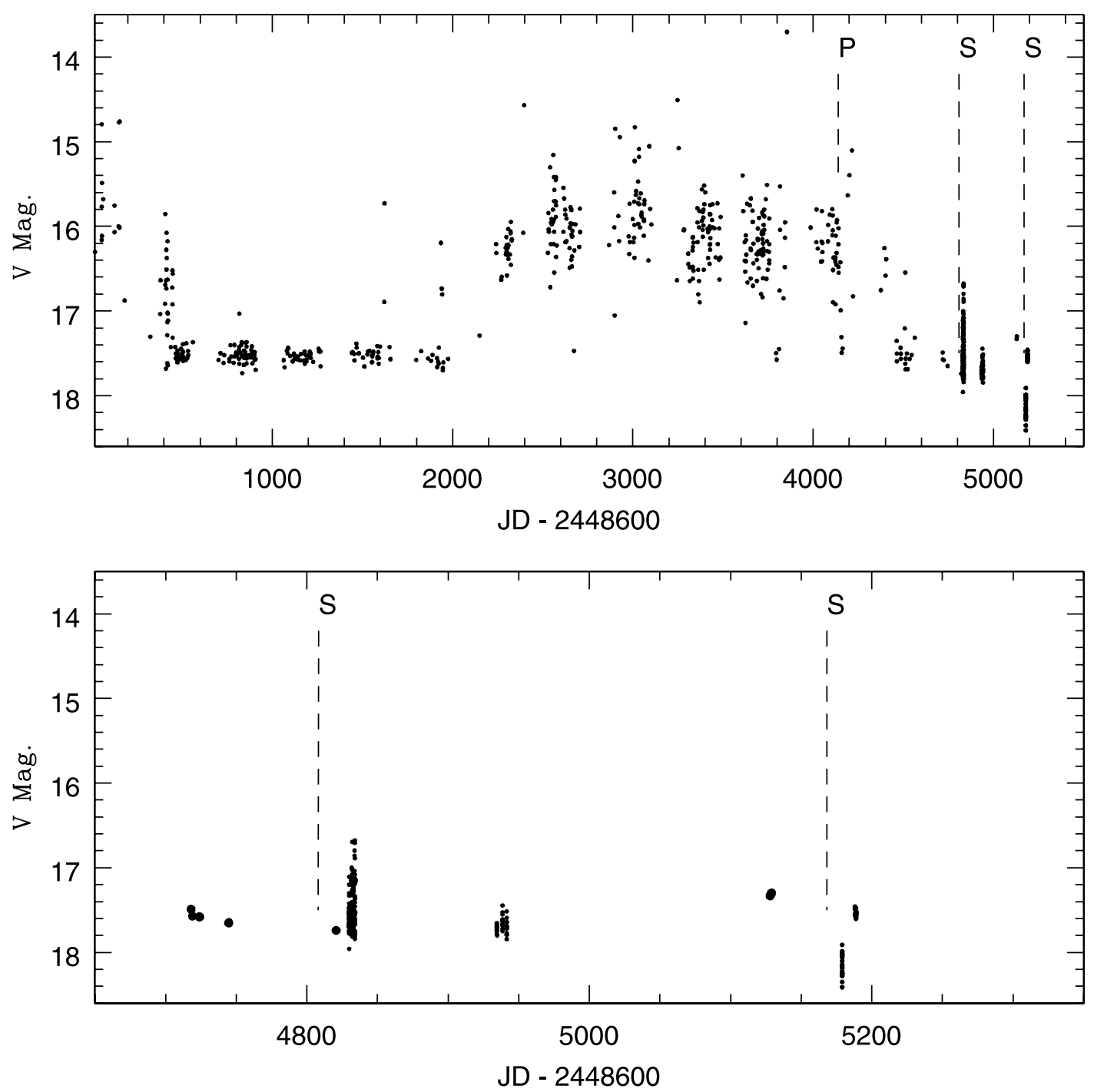

FIG. 6.- - Long-term light curves of ST LMi. Top: $V$-band photometry following ST LMi from late 1991 to early 2006. Bottom: Zoom-in on the year surrounding our IRTF SPEX spectroscopy. The P denotes the epoch of our photometry, while $\mathrm{S}$ marks our spectroscopic data during the 2005 extreme low state and the 2006 low state, respectively. 


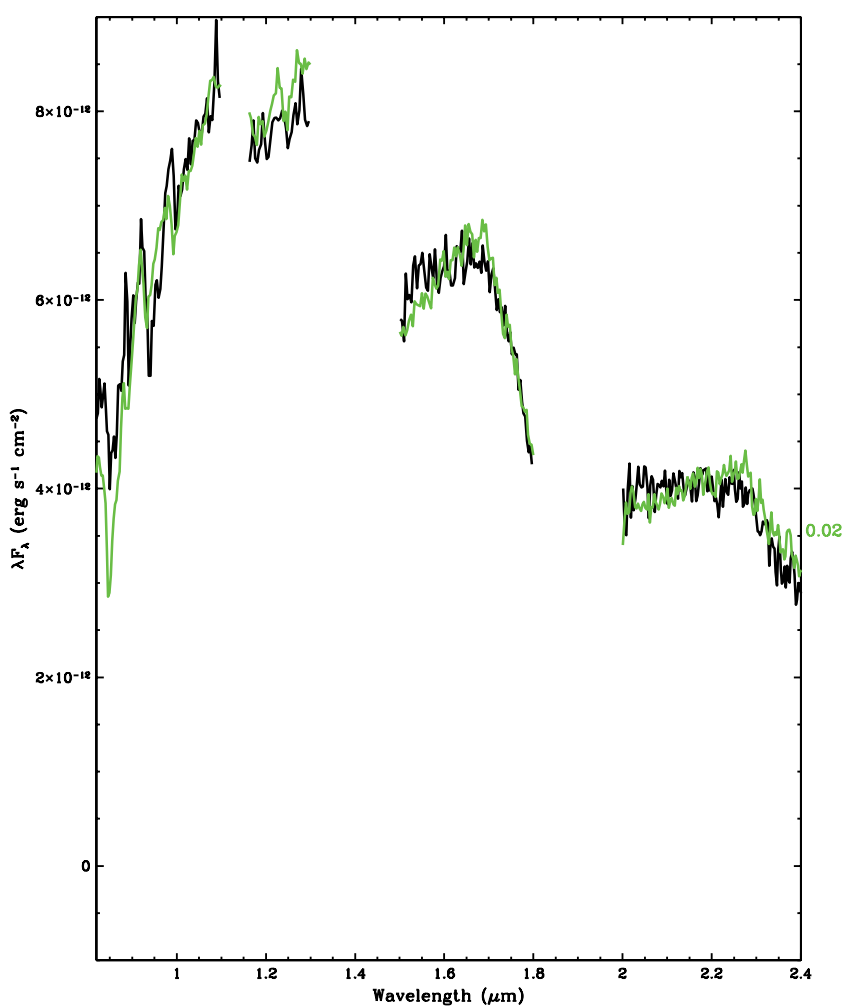

FIG. 7.- IRTF data of ST LMi in an extreme low state (2005). An M6 template spectrum is plotted during the dim phase $(\phi=0.02)$ confirming the spectral classification.

two epochs agree to within their errors, the same cannot be said for both the temperatures and values of $\log \Lambda$. Our SPEX data show a system with a cooler plasma than that inferred by BFW91. To quantify this difference, we evoke the results of Fischer \& Beuermann (2001), who found $k T_{\max } \propto \dot{m} B^{-2.6}$, which we rewrite as $\dot{m}_{1} / \dot{m}_{2}=\left(k T_{1} / k T_{2}\right)\left(B_{1} / B_{2}\right)^{2.6}$. Plugging in the average bright-phase parameters from the two epochs shows that the $\dot{m}$ for the modeled SPEX data must be a factor of 2.34 lower than that active during the BFW91 observations. The higher temperatures found in BFW91 may be an artifact of medianing together moving cyclotron harmonics over $\sim 40 \%$ of the orbit, which artifically broadens each feature.

The final spectra and models allow us to understand the changing morphology of AM Her's NIR light curves. In Figure $3 b$ the $J$ band is well explained by ellipsoidal variations alone, while in the $H+K$ bands the cyclotron emission component is substantial; with a maximum contribution of 0.25 mag in both bands, it disappears at $\phi=0.00$. The $K$-band cyclotron component is relatively constant over the phases $0.75 \leq \phi \leq 0.25$. Such behavior is explained by the cyclotron models shown in Figure 4. Near $\phi=0.00$ the $n=4$ harmonic dominates due to the low viewing angle $\left(\Theta \simeq 35^{\circ}\right)$ at that time. Consequently, no emission is seen in the $H$ band. Later $(0.25 \leq \phi \leq 0.75)$ the viewing angle is larger, causing the higher harmonics ( $n=5$ and 6 ) to be excited and thus a peak in the $H$-band emission is observed. Because the $n=4$ harmonic is mostly optically thick, however, the cyclotron emission in the $K$ band remains relatively constant over the entire bright phase.

The simultaneous optical photometry is also interesting. In the $R$ and $I$ bands the data were entirely explained by the ellipsoidal models alone for the first full orbit of phase coverage, in line with expectations from our 13.7 MG cyclotron models that have very little emission shortward of $1.2 \mu \mathrm{m}$. Subsequently, a brightening event seems to have occurred, with $\Delta m=0.20$ in both bands. The $B$ and $V$ bands are more complex and cannot be explained by ellipsoidal models alone. Additional modulation was observed at the level of $\Delta m=0.22$ and 0.08 for $B$ and $V$, respectively. Gänsicke et al. (1998) modeled similar UV light curves as a hot spot. In that work, three UV bands covering wavelength regions of 1150-1167, 1254-1286, and 1412-1427 $\AA$ were found to be consistent with a $47,000 \mathrm{~K}$ hotspot centered on $\psi=0^{\circ}$ and covering $9 \%$ of the WD surface. We find the similarity of our $B$-band light curve to Gänsicke et al.'s UV light curves to be striking. Both light curves show identical phasing and have amplitudes that are consistent. We note, however, the large value of $B-V \simeq 0.15$ during the bright phase. For any reasonable hotspot, $B-V$ should be closer to 0.00 . Because (1) the UV light curves predict a very similar geometry ( $i, \beta$, and phase) to our cyclotron emission region and (2) the limited wavelength coverage of the UV light curves, we find that this excess emission could be caused by a partially saturated high-field cyclotron harmonic ( $n=3$ or 4 ) that falls off toward the blue end of the $V$ band and extends through the bluest UV band. Such a broad harmonic $(\Delta \lambda=0.4 \mu \mathrm{m})$ is expected in cyclotron emission (see AM Her's $n=4$ harmonic in Fig. 4 , which is more or less flat from 1.9 to $2.4 \mu \mathrm{m}$ ). If the emission were from the $n=4$ harmonic it would imply a $\sim 90 \mathrm{MG}$ field. We also speculated on the presence of a similar secondary high-field pole for EF Eri in Paper I.

During the bright phase of the 2006 low state, ST LMi displayed cyclotron emission with the following properties: $B=$ $12.1 \pm 0.5 \mathrm{MG}, k T=3.3 \pm 1.8 \mathrm{keV}$, and $\log \Lambda=5.7 \pm 0.6$ similar to the cool spot found in FBW93. In addition, our accretion region appears to be in a similar location on the WD surface: at magnetic longitude $\psi=120^{\circ}$, lagging behind the onset of the secondary accretion region found in FBW93 by $\Delta \phi \simeq$ 0.13 , likely due to the accumulation in phase-error between 1991 and 2005. Unfortunately, no errors are given for any of the cyclotron parameters in FBW93 and thus the significance of the difference in results is hard to assess. However, both the FBW93 magnetic field strength and the plasma temperature agree to within our errors. More interesting is the nondetection of their primary accretion region which should trail the observed "secondary" emission region by about 0.10 in phase, implying an onset at $\phi \simeq 0.60$, which is not seen.

\subsection{Ellipsoidal versus Cyclotron-derived Inclinations}

In both cases the geometry of the emission region was consistent with a simple single-spot model with a constant orbital inclination and magnetic colatitude. In Figure 9 we plot (black) the cyclotron derived values of the viewing angle against the orbital phase for both AM Her (top) and ST LMi (bottom). In red are the simple geometrical models, with the blue shading indicating phases for which the cyclotron regions are self eclipsed. The models fit the AM Her data well for nearly all phases, only deviating near self-eclipse ingress and egress, where the viewing angle is changing rapidly compared to the cadence of our spectroscopy. For ST LMi, the bright phase is relatively short, lasting $\simeq 40 \%$ of the orbit. Consequently, few data were available to constrain its geometry. We thus used published values of $i$ and $\beta$ for an additional constraint, finding that we could match the data with models quite similar to those found in the literature.

For AM Her, the cyclotron models imply an orbital inclination of $i=50^{\circ}$, identical to that found in the ellipsoidal modeling effort, although higher angles are possible if additional sources of dilution remain in the IR light curves. Agreement was also found for the ellipsoidal and cyclotron inclinations for ST LMi, with 


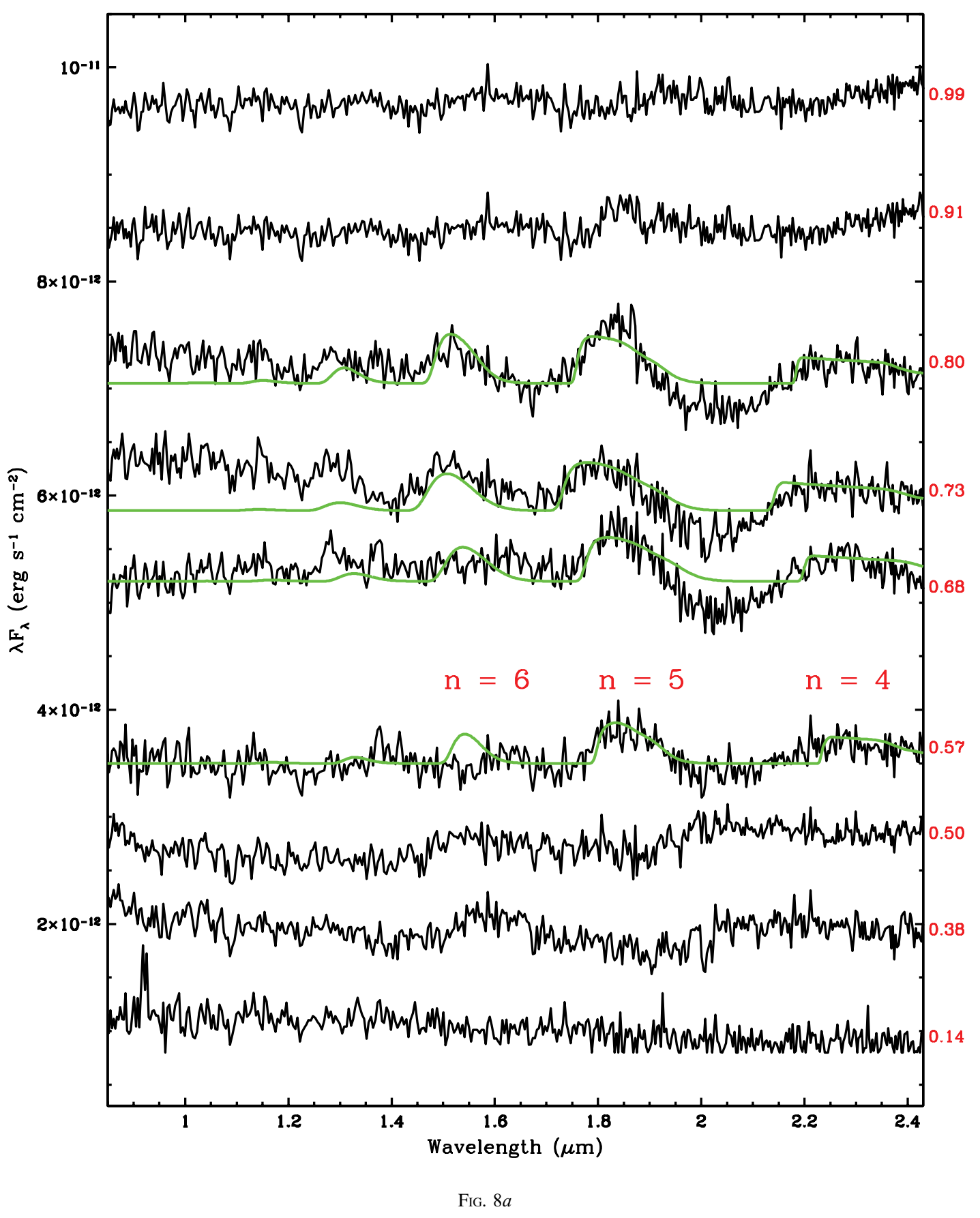

FIG. 8.-(a) Phase-resolved SE-subtracted spectroscopy of ST LMi, taken in 2006 February during a low state. The IRTF SPEX data are plotted (black) as a stacked series-a constant increment of $\lambda F_{2}=1.2 \times 10^{-12} \mathrm{ergs} \mathrm{s}^{-1} \mathrm{~cm}^{-2}$ is added to each spectrum to offset it from the spectrum below it. The SE spectrum subtracted from each phase was a median of three dim-phase spectra. The best-fit cyclotron model for each of the bright-phase spectra are shown in green. (b) Same as $(a)$, but for the 2005 February extreme low state. 


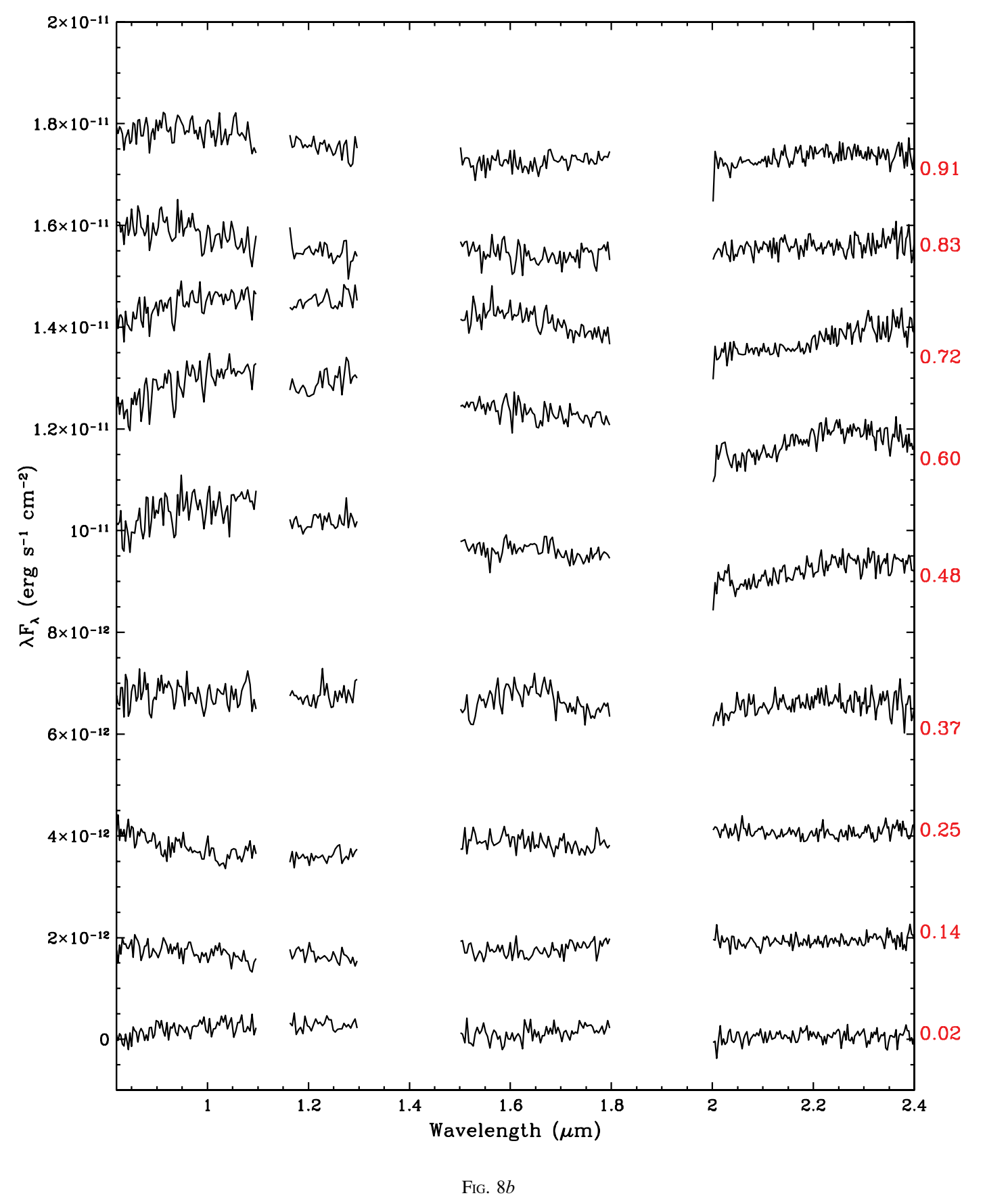


TABLE 3

Cyclotron Modeling Parameters for ST CrB

\begin{tabular}{|c|c|c|c|c|c|}
\hline Phase & $\begin{array}{c}B \\
(\mathrm{MG})\end{array}$ & $\begin{array}{c}T \\
(\mathrm{keV})\end{array}$ & $\theta$ & $\log \Lambda$ & $\chi_{\nu}^{2}$ \\
\hline $0.14 \ldots \ldots \ldots \ldots \ldots$ & $\ldots$ & $\ldots$ & $\ldots$ & $\ldots$ & $\ldots$ \\
\hline $0.26 \ldots \ldots \ldots \ldots \ldots$ & $\ldots$ & $\ldots$ & $\ldots$ & $\ldots$ & $\ldots$ \\
\hline $0.38 \ldots \ldots \ldots \ldots \ldots$ & $\ldots$ & $\ldots$ & $\ldots$ & $\ldots$ & $\ldots$ \\
\hline $0.50 \ldots \ldots \ldots \ldots \ldots$ & $\ldots$ & $\ldots$ & $\ldots$ & $\ldots$ & $\ldots$ \\
\hline $0.57 \ldots \ldots \ldots \ldots \ldots$ & 12.0 & 3.2 & 85.0 & 5.5 & 2.19 \\
\hline $0.68 \ldots \ldots \ldots \ldots \ldots$ & 12.0 & 3.2 & 78.0 & 5.5 & 2.61 \\
\hline $0.73 \ldots \ldots \ldots \ldots \ldots$ & 12.2 & 3.4 & 75.0 & 6.1 & 3.75 \\
\hline $0.85 \ldots \ldots \ldots \ldots \ldots$ & 12.2 & 3.4 & 82.0 & 5.9 & 2.24 \\
\hline $0.91 \ldots \ldots \ldots \ldots \ldots$ & $\ldots$ & $\cdots$ & $\cdots$ & $\ldots$ & $\cdots$ \\
\hline $0.99 \ldots \ldots \ldots \ldots \ldots$ & $\ldots$ & $\ldots$ & $\ldots$ & $\ldots$ & $\ldots$ \\
\hline
\end{tabular}

values of $i=55^{\circ}$ and $40^{\circ}$, respectively. The later value represents the lowest inclination ellipsoidal model, and values of $i=$ $55^{\circ}$ are more consistent with the light curve (see Fig. 3). Some caution, however, should be given to the fact that the spectroscopy and light curves of ST LMi were taken at different epochs when the object was in different states, which could affect the accretion geometry. In Schwope et al. (1993) the authors found that the polar MR Serpentis appeared to show longitudinal migration of the accretion spot by $\simeq 30^{\circ}$, as well as a $10^{\circ}$ shift in the magnetic colatitude between its high and low states.

\subsection{ST LMi in an Extreme Low State}

In Figure $8 b$ we present SE-subtracted SPEX data obtained during our 2005 February 7 observing run, which show a conspicuous lack of cyclotron emission throughout the entire orbital cycle. The extreme low state of ST LMi is corroborated by nearepoch (2006 February 12) optical light curves obtained with the WIYN $0.9 \mathrm{~m}$, showing the system in a deep low state. Despite the poor telluric correction (the spectra were faint), the only strong feature in the bright phase $(0.50 \leq \phi \leq 0.85)$ is small bump longward of $2.2 \mu \mathrm{m}$, caused by under subtraction of the secondary star at those phases. In this series of papers (see Papers I, II, and Szkody et al. 2008) we have modeled seven polars, representing $\sim 10 \%$ of all known mCVs. Included in this sample were EQ Cet, the prototype low-accretion rate polar, MQ Dra (=SDSS 1553), the prototype prepolar, and EF Eri, well known for its protracted low state. Intriguingly, in each of these objects strong cyclotron emission was observed, while the extreme low state data set of ST LMi is the only example in which cyclotron emission completely disappeared. A similar situation probably explains the 2006 February 12 photometric low state found by Kafka et al. (2007). This suggests that normal polars can have periods that appear completely devoid of detectable accretion.

\section{CONCLUSION}

We obtained a full orbit of phase-resolved IR spectra for both AM Her and ST LMi. We found both objects to be dominated by emission from the secondary in the IR. To remove this compo-

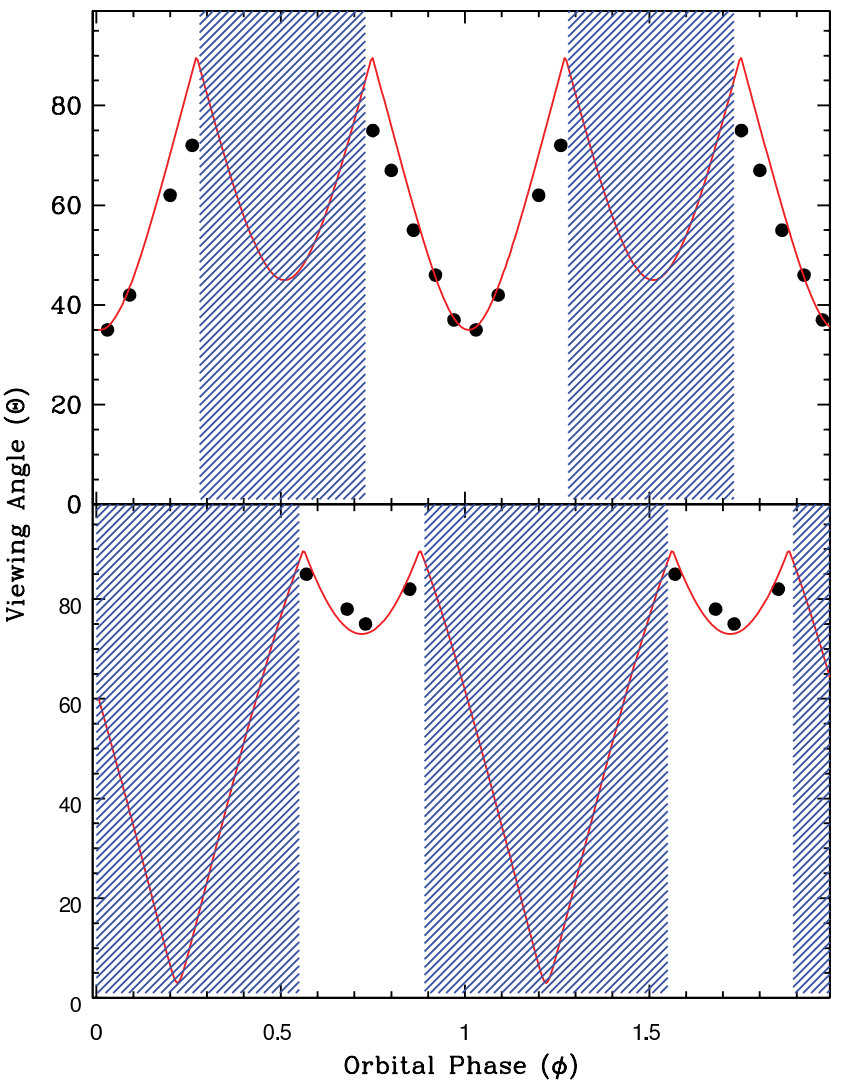

FIG. 9.-Top: Derived value of the viewing angle $(\Theta)$ for AM Her is plotted vs. the orbital phase $(\phi)$ in black, with the best-fit geometry $\left(i=50^{\circ}, \beta=85^{\circ}\right)$ overlaid in red. The blue shading indicates phases for which AM Her is selfeclipsed. Bottom: Same as top, but for ST LMi and with a geometrical model of $\left(i=55^{\circ}, \beta=128^{\circ}\right)$.

nent, we utilized the fact that emission regions for both stars were self-eclipsed. Thus, at each phase we subtract a dim-phase or stream-emission spectrum. Because of the changing spectral type of the secondary, we found that medianing dim-phase spectra over $\simeq 25 \%$ of an orbit produced a better subtraction. For AM Her, we found a phase averaged model of $B=13.6_{-0.8}^{+1.0} \mathrm{MG}$, $k T=4.0_{-1.0}^{+1.5} \mathrm{keV}$ (see Table 2 for specifics at each phase). In addition, we found that the viewing angle varied in a manner consistent with expectations from a system with $i=50^{\circ}$ and $\beta=85^{\circ}$. For ST LMi, we collected two data sets. The first had ST LMi in a low state with $V \simeq 17.4$ and displayed weak cyclotron harmonics that were difficult to decouple from the water vapor signatures leftover after SE-subtraction. We found a phase averaged model of $B=12.1 \pm 0.5 \mathrm{MG}, k T=3.3 \pm 1.8 \mathrm{keV}$ in an accretion region consistent with $i=55^{\circ}$ and $\beta=128^{\circ}$. For ST LMi, we include a second data set, taken when the object was in an extreme low state showing no substantial cyclotron emission. The nondetection of cyclotron emission contrasts with our earlier data from both EQ Cet and MQ Dra both of which show cyclotron emission, even while being in extreme low states.

\section{REFERENCES}

Araujo-Betancor, S., Gänsicke, B. T., Long, K. S., Beuermann, K., de Martino, D., Sion, E. M., \& Szkody, P. 2005, ApJ, 622, 589

Bailey, J., Ferrario, L., \& Wickramasinghe, D. T. 1991, MNRAS, 251, P37

Brainerd, J. J., \& Lamb, D. Q. 1985, Cataclysmic Variables and Low-Mass X-Ray Binaries, ed. D. Q. Lamb \& J. Patterson (Dordrecht: Reidel), 247

Campbell, R. K., Harrison, T. E., Mason, E., Howell, S. B., \& Schwope, A. D. 2008a, ApJ, 678, 1304 (Paper II)
Campbell, R. K., Harrison, T. E., Schwope, A. D., \& Howell, S. B. 2008b, ApJ, 672, 531 (Paper I)

Ciardi, D., Howell, S. B., Dhillon, V. S., Wagner, R. M., Hauschildt, P. H., \& Allard, F. 1998, PASP, 110, 1007

Cropper, M. 1986, MNRAS, 222, 853

Cropper, M., Ramsay, G., \& Wu, K. 1998, MNRAS, 293, 222

Davey, S., \& Smith, R. C. 1992, MNRAS, 257, 476 
Ellis, T., et al. 1992, Proc. SPIE, 1765, 94

Ferrario, L., Bailey, J., \& Wickramasinghe, D. T. 1993, MNRAS, 262, 285

Fischer, A., \& Beuermann, K. 2001, A\&A, 373, 211

Gänsicke, B. T., Fischer, A., Silvotti, R., \& de Martino, D. 2001, A\&A, 372, 557

Gänsicke, B. T., Hoard, D. W., Beuermann, K., Sion, E. M., Szkody, P. 1998, A\&A, 338, 933

Gänsicke, B. T., Long, K. S., Barstow, M. A., \& Hubeny, I. 2006, ApJ, 639, 1039

Harrison, T. E., Howell, S. B., Huber, M. E., Osborne, H. L., Holtzman, J. A., Cash, J. L., \& Gelino, D. M. 2003, AJ, 125, 2609

Harrison, T. E., Howell, S. B., Szkody, P., \& Cordova, F. 2005, ApJ, 632, L123

Howell, S. B., Ciardi, D. R., Dhillon, V. S., \& Skidmore, W. 2000, ApJ, 530, 904

Hubeny, I., \& Lanz, T. 1995, ApJ, 439, 875

Kafka, S., \& Honeycutt, R. K. 2005, AJ, 130, 742

Kafka, S., \& Honeycutt, R. K., Howell, S. B., \& Harrison, T. E. 2005, AJ, 130,2852

Kafka, S., Howell, S. B., Honeycutt, R. K., \& Robertson, J. W. 2007, AJ, 133, 1645

Knigge, C. 2006, MNRAS, 373, 484

Peacock, T., Cropper, M., Bailey, J., Hough, J. H., \& Wickramasinghe, D. T. 1992, MNRAS, 259, 583

Potter, S. B. 2000, MNRAS, 314, 672
Ramsay, G., Cropper, M., Wu, K., Mason, K. O., Cordova, F. A., \& Priedhorsky, W. 2004, MNRAS, 350, 1373

Rayner, J. T., Toomey, D. W., Onaka, P. M., Denault, A. J., Stahlberger, W. E., Vacca, W. D., Cushing, M. C., \& Wang, S. 2003, PASP, 115, 362

Schmidt, G. D., Stockman, H. S., \& Grandi, S. A. 1983, ApJ, 271, 735

Schwope, A. D. 1990, Rev. Mod. Astron., 3, 44

Schwope, A. D., Beuermann, K., Jordan, S., \& Thomas, H.-C. 1993, A\&A, 278,487

Schwope, A. D., Brunner, H., Buckley, D., Greiner, J., Heyden, K. v. d., Neizvestny, S., Potter, S., \& Schwarz, R. 2002, A\&A, 396, 895

Sion, E. 1999, PASP, 111, 532

Sirk, M. M., \& Howell, S. B. 1998, ApJ, 506, 824

Stockman, H. S., Foltz, C. B., Schmidt, G. D., \& Tapia, S. 1983, ApJ, 271, 725

Szkody, P., Linnell, A. P., Campbell, R. K., Plotkin, R. M., Harrison, T. E., Holtzman, J., Seibert, M., \& Howell, S. B. 2008, ApJ, in press

Tapia, S. 1977, ApJ, 212, L125

Thorstensen, J. R. 2003, AJ, 126, 3017

Vacca, W. D., Cushing, M. C., \& Rayner, J. T. 2003, PASP, 115, 389

Warner, B. 1995, Ap\&SS, 232, 89

Watson, C. A., Dhillon, V. S., Rutten, R. G. M., \& Schwope, A. D. 2003, MNRAS, 341, 129

Young, P., Schneider, D. P., \& Shectman, S. A. 1981, ApJ, 245, 1043 